\title{
Classification of Fricatives Using Novel Modulation Spectrogram Based Features
}

\author{
Kewal D. Malde, Anshu Chittora, and Hemant A. Patil \\ Dhirubhai Ambani Institute of Information and Communication Technology, \\ Gandhinagar, Gujarat, India \\ \{kewal_dhiraj, anshu_chittora, hemant_patil\}@daiict.ac.in
}

\begin{abstract}
In this paper, we propose the use of a novel feature set, i.e., modulation spectrogram for fricative classification. Modulation spectrogram gives 2-dimensional (i.e., 2-D) feature vector for each phoneme. Higher Order Singular Value Decomposition (HOSVD) is used to reduce the size of large dimensional feature vector obtained by modulation spectrogram. These features are then used to classify the fricatives in five broad classes on the basis of place of articulation (viz., labiodental, dental, alveolar, post-alveolar and glottal). Four-fold cross-validation experiments have been conducted on TIMIT database. Our experimental results show $89.09 \%$ and $87.51 \%$ accuracies for recognition of place of articulation of fricatives and phoneme-level fricative classification, respectively, using 3-nearest neighbor classifier.
\end{abstract}

Keywords: Fricative classification, modulation spectrogram, HOSVD, place of articulation, acoustic frequency and modulation frequency.

\section{Introduction}

The main purpose of this paper is to use modulation spectrogram-based features to distinguish between various fricatives sounds extracted manually from TIMIT database. Consonants are classified in three broad classes based on the manner of articulation, viz., stops, affricates and fricatives. Fricatives class is the largest class of consonants in TIMIT database. In total, there are 10 fricatives divided in five broad classes based on place of articulation, viz., labiodental $(/ f /, / v /)$, dental $(/ t h /, / d h /)$, alveolar $(/ \mathrm{s} /, / z /)$, post-alveolar $(/ \mathrm{sh} /, / / \mathrm{h} /)$ ) and glottal $(/ \mathrm{h} h /, / \mathrm{hv} /)$. Fricatives can also be classified as unvoiced and voiced on the basis of voicing activity, i.e., relaxed or vibrating vocal folds. From all the examples of the fricatives mentioned above in pairs, the former in each pair falls under unvoiced fricatives while later falls under voiced fricatives [1-4].

Fricatives are generated by a narrow constriction in the vocal tract, giving rise to a steady frication noise. The degree of constriction also plays an important role in spectral characteristics (as secondary effect with vocal tract being the primary one). Place of articulation and the spectral characteristics are influenced on the basis of location of constriction by the tongue (at the back, center, or front of the oral tract, as well as at the teeth or lips) [1]. 
Prior work on fricative classification on TIMIT database is based on the knowledge-based, acoustic-phonetic features where Seneff's auditory model is used as front-end processing. An accuracy of $87 \%$ was obtained for the overall classification of fricatives (on training data set) [5-7].

In this paper, we introduce the use of 2-D representation of modulation spectrogram based for classification of fricatives. Modulation spectrogram-based feature set integrates the concept of sensory perception with signal processing methodology to achieve a significant improvement in the representation and coding of acoustic signals [8-9]. Recently, modulation spectrogram has been used in voice quality classification, pathology classification and speech recognition [10].

\section{Modulation Spectrogram}

Modulation spectrogram is the visual representation of the spectrum of the combination of acoustic and modulation frequencies in a speech segment. The spectrogram is square of the magnitude of short-time Fourier transform (STFT) of speech signal, representing the energy distribution of the signal w.r.t. time and acoustic frequency parameters. Modulation spectrogram is the spectrum of magnitude spectra of speech segment, representing energy distribution of the signal w.r.t. acoustic and modulation frequency parameters. Each phoneme has different combination of acoustic and modulation frequencies (depending on the constriction formed by the tongue). This motivated us to use modulation spectrogram for fricative classification. Modulation spectrogram is given by [10],

$$
\begin{gathered}
X_{m}(k)=\sum_{n=-\infty}^{\infty} h(m M-n) x(n) W_{I_{A}}^{k n}, \\
X_{l}(k, i)=\sum_{m=-\infty}^{\infty} g(l L-m)\left|X_{m}(k)\right| W_{I_{M}}^{i m},
\end{gathered}
$$

where $k=0,1, \ldots,\left(I_{A}-1\right)$ is acoustic frequency, $W_{I_{A}}=e^{-j\left(2 \pi / I_{A}\right)}, W_{I_{M}}=e^{-j\left(2 \pi / I_{M}\right)}$, $i=0,1, \ldots,\left(I_{M}-1\right)$ is modulation frequency; $n$ is the sample index in time-domain, $m$ is the frame index in equation (1) and $l$ is the frame index in equation $(2) ; h(n)$ and $g(m)$ are the analysis window for acoustic and modulation frequency with hop sizes $M$ and $L$, respectively. Here, $\left|X_{l}(k, i)\right|^{2}$ represents the modulation spectrogram of signal $x(n)$.

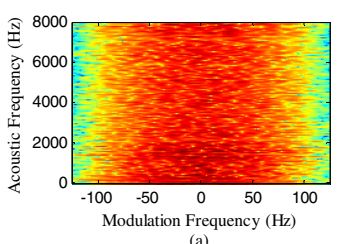

(a)

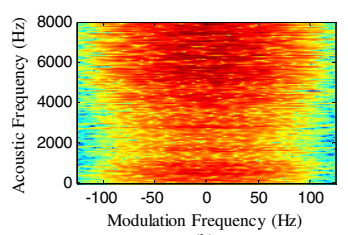

(b)

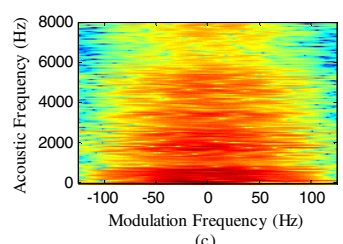

(c)

Fig. 1. Modulation Spectrogram: (a) labiodental /f/, (b) alveolar /s/, (c) glottal $/ \mathrm{hh} /$ 
From some of the examples of modulation spectrogram shown in Fig. 1, we can observe that phonemes from different classes have their energy distributed across different regions of acoustic frequency $\left(f_{a}\right)$ and modulation frequency $\left(f_{m}\right)$ (as the mode of constriction formed by the tongue is different for each phoneme); hence, it can serve as a potential cue for classification of fricative sounds.

\section{Higher Order Singular Value Decomposition (HOSVD)}

Higher Order Singular Value Decomposition (HOSVD) is applied to 3- $D$ feature set. HOSVD theorem is used for dimension reduction of a 3-D tensor as described in [11]. First of all, modulation spectrogram which is a $2-D$ feature vector is obtained for each phoneme segment. Then a 3-D tensor is formed by stacking 2-D modulation spectrogram feature vector (i.e., $B \in \mathbb{R}^{I_{A} \times I_{M}}$ by considering only positive modulation frequencies taking symmetric nature of modulation spectrogram into consideration) for all phoneme samples ( say $I_{S}$ ) under each class. Thus, the tensor $A \in \mathbb{R}^{I_{A} \times I_{M} \times I_{S}}$ can be represented in HOSVD form as:

$$
A=S \times_{1} U_{A} \times_{2} U_{M} \times_{3} U_{S},
$$

where $S$ is the core tensor with same dimension as $A . U_{A} \in \mathbb{R}^{I_{A} \times I_{A}}, U_{M} \in \mathbb{R}^{I_{M} \times I_{M}}$ and $U_{S} \in \mathbb{R}^{I_{S} \times I_{S}}$ are the unitary matrices of the corresponding subspaces of $I_{A}, I_{M}$ and $I_{S}$. The matrices $U_{A}$ and $U_{M}$ can be obtained by unfolding tensor $A$. $U_{A}$ and $U_{M}$ are obtained from SVD representation of the unfolded matrices $A_{A} \in \mathbb{R}^{I_{M} \times I_{A} I_{S}}$ and $A_{M} \in \mathbb{R}^{I_{A} \times I_{M} I_{S}}$. Then, $\hat{U}_{A} \in \mathbb{R}^{I_{A} \times R_{A}}$ and $\hat{U}_{M} \in \mathbb{R}^{I_{M} \times R_{M}}$ are obtained from these unitary matrices $U_{A}$ and $U_{M}$, by retaining only first $R_{A}$ and $R_{M}$ vectors, respectively. Now, using these obtained $\hat{U}_{A}$ and $\hat{U}_{M}$, we get the reduced tensor as,

$$
\hat{A}=S \times_{1} \hat{U}_{A} \times_{2} \hat{U}_{M} \times_{3} U_{S} .
$$

And finally un-stacking the reduced tensor $\hat{A}$, reduced feature vector $K \in \mathbb{R}^{R_{A} \times R_{M}}$ (per phoneme) is obtained as,

$$
K=B \times_{1} \hat{U}_{A}^{T} \times_{2} \hat{U}_{M}^{T}=\hat{U}_{A}^{T} \cdot B \cdot \hat{U}_{M} .
$$

\section{Experimental Setup}

\subsection{Database Used}

TIMIT database contains a total of 6300 sentences, 10 sentences spoken by each of 630 speakers from 8 major dialect regions of the United States. In addition to a speech waveform (i.e., *.wav) file, TIMIT corpus includes three associated transcription files (viz., *.txt, *.wrd, *.phn). With the help of phonetic transcription (i.e., *.phn) file we 
obtained fricative segments. Out of all obtained fricative segments, we randomly selected 1000 samples of each phoneme (from both training and testing dataset) under fricative class. In our work, we have considered all 10 fricatives.

\subsection{Feature Extraction}

First of all, find the modulation spectrogram for each phoneme using COG (center of gravity) method [12]. In modulation spectrogram, Y-axis represents acoustic frequency which ranges from 0 to $F_{s} / 2$ (here, $F_{s}=16 \mathrm{kHz}$ ) (equally spaced in 54 bins) and $\mathrm{X}$-axis represents modulation frequency which ranges from $-100 \mathrm{~Hz}$ to $+100 \mathrm{~Hz}$ (equally spaced bins). The modulation frequency dimension varies with respect to phoneme class and phoneme duration. For making the classification task easier, $f_{m}$ dimension is fixed to 140 by zero padding. Since the modulation spectrogram is symmetric around $f_{m}=0$, only positive modulation frequencies is considered for analysis. Using the symmetry property of modulation spectrogram the feature size is reduced from $54 \times 140$ to $54 \times 71$. To further reduce the feature dimension, HOSVD theorem is applied which gives a feature vector of dimension $f_{a} \times f_{m}$.

\section{$5 \quad$ Experimental Results}

Four-fold cross-validation experiments have been conducted on 56 different combinations of $f_{a}$ and $f_{m}$. We have considered following values of $f_{a}$ and $f_{m}, v i z ., f_{a}=$

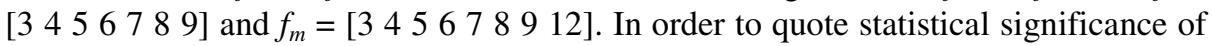
our experimental results, complete phoneme set is randomly divided into training and testing datasets (as per training-testing ratio), for every experiment.

Results of 4 -fold cross-validation of recognition of place of articulation of fricatives and phoneme-level fricative classification with feature vector dimension of $3 \times 3$ and 75:25\% training-testing ratios are shown in Table 1 and Table 2, respectively. Fig. 2 shows the effect on classification accuracy due to change in acoustic $\left(f_{a}\right)$ and modulation $\left(f_{m}\right)$ frequencies. From Fig. 2, we can see that initially accuracy increases as the value $f_{a}$ and $f_{m}$ is increased (as the phoneme are more closely related in spectral characteristics, we need relatively higher dimension of feature vector for better classification of data), however, after certain amount of increase in the values of $f_{a}$ and $f_{m}$, any further increase in the value decreases the classification accuracy (as it introduces more amount of redundancies). It can also be observed that for lower value of $f_{m}$ (i.e., $f_{m}=3$ and $f_{m}=4$ ) accuracy is almost constant. We can also infer that change in the value of $f_{m}$ affects the accuracy more significantly as compared to the change in the value of $f_{a}$. Best classification accuracy is obtained for feature of dimension $3 \times 8$. However, we have selected the optimum feature vector dimension as $3 \times 3$ since the improvement in the accuracy is approximately $1 \%$ while the dimension is reduced by a factor of approximately 2.67. The reduction in feature vector dimension is advantageous in reducing computational complexity and computation time. 


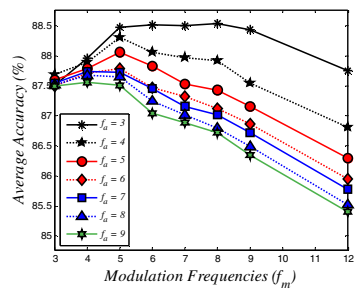

(a)

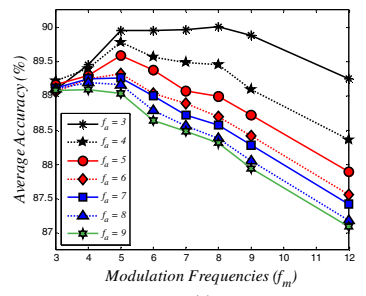

(c)

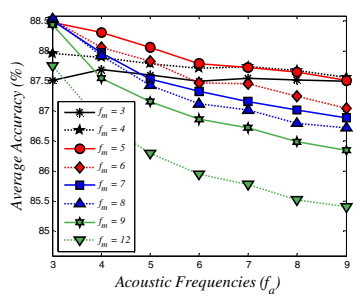

(b)

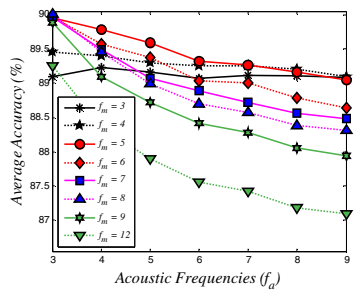

(d)

Fig. 2. Average accuracy (in \%) for fricative consonant classification at phoneme-level: Effect on classification accuracy due to change in (a) modulation frequency $\left(f_{m}\right)$, (b) acoustic frequency $\left(f_{a}\right)$; Average accuracy (in $\%$ ) for recognition of place of articulation of fricatives: Effect on recognition accuracy due to change in (c) modulation frequency $\left(f_{m}\right)$, (d) acoustic frequency $\left(f_{a}\right)$. In all sub-figures, units of acoustic and modulation frequencies are in bins.

Table 1. Confusion matrix for accuracy (in \%) for recognition of place of articulation of fricatives. Average accuracy is $89.09 \%$.

\begin{tabular}{|c|c|c|c|c|c|c|c|}
\hline & \multicolumn{5}{|c|}{ Detected } \\
\hline & & & Labiodental & Dental & Alveolar & Post-alveolar & Glottal \\
\hline & & & $|f|, / v /$ & $/ t h /, / d h /$ & $/ s /, \mid z /$ & $/ \mathrm{sh} /, / \mathrm{zh} /$ & $/ h h /, / h v /$ \\
\hline \multirow{5}{*}{$\begin{array}{l}\bar{\pi} \\
\text { Z्र } \\
\text { \& }\end{array}$} & Labiodental & $/ f /, / v /$ & 88.22 & 3.43 & 2.97 & 1.97 & 3.41 \\
\hline & Dental & $/ t h /, / d h /$ & 3.45 & 89.85 & 2.34 & 1.41 & 2.94 \\
\hline & Alveolar & $|s /| z /$, & 2.92 & 2.34 & 88.27 & 3.44 & 3.03 \\
\hline & Post-alveolar & $/ \mathrm{sh} /, / z h /$ & 2.01 & 1.37 & 3.48 & 90.81 & 2.33 \\
\hline & Glottal & $/ h h /, / h v /$ & 3.43 & 2.97 & 2.98 & 2.32 & 88.30 \\
\hline
\end{tabular}

Table 2. Confusion matrix for phoneme-level fricative classification accuracy (in \%). Average accuracy is $87.51 \%$.

\begin{tabular}{|c|c|c|c|c|c|c|c|c|c|c|c|}
\hline & \multicolumn{10}{|c|}{ Detected } \\
\hline & & $|f|$ & $/ v /$ & $/ \mathrm{th} /$ & $/ d h /$ & $/ s /$ & $|z|$ & $/ \mathrm{sh} /$ & $/ z h /$ & $/ \mathrm{hh} /$ & $/ h v /$ \\
\hline \multirow{10}{*}{ 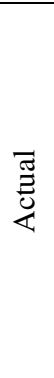 } & $|f|$ & 85.89 & 1.64 & 2.71 & 1.13 & 1.67 & 1.75 & 1.50 & 0.57 & 1.91 & 1.23 \\
\hline & $/ v /$ & 1.61 & 87.30 & 1.65 & 1.37 & 1.22 & 1.29 & 1.29 & 0.58 & 1.78 & 1.90 \\
\hline & $/ t h /$ & 2.73 & 1.68 & 85.70 & 1.30 & 1.63 & 1.63 & 1.36 & 0.44 & 2.02 & 1.51 \\
\hline & $/ d h /$ & 1.08 & 1.42 & 1.32 & 91.37 & 0.70 & 0.73 & 0.75 & 0.27 & 1.26 & 1.10 \\
\hline & $/ s /$ & 1.63 & 1.24 & 1.55 & 0.71 & 86.21 & 2.10 & 2.66 & 1.01 & 1.44 & 1.45 \\
\hline & $|z|$ & 1.67 & 1.30 & 1.64 & 0.79 & 2.08 & 86.15 & 2.28 & 0.94 & 1.48 & 1.68 \\
\hline & $/ s h /$ & 1.53 & 1.37 & 1.37 & 0.70 & 2.71 & 2.24 & 85.39 & 1.24 & 1.49 & 1.95 \\
\hline & $/ z h /$ & 0.58 & 0.55 & 0.43 & 0.23 & 0.98 & 1.03 & 1.21 & 93.77 & 0.52 & 0.71 \\
\hline & $/ h h /$ & 1.93 & 1.78 & 2.11 & 1.24 & 1.39 & 1.50 & 1.44 & 0.54 & 86.48 & 1.59 \\
\hline & $/ h v /$ & 1.28 & 1.87 & 1.43 & 1.16 & 1.41 & 1.67 & 1.96 & 0.70 & 1.73 & 86.80 \\
\hline
\end{tabular}




\section{Summary and Conclusions}

In this paper, we have proposed the use of modulation spectrogram-based features using a simple classifier, i.e., 3-nearest neighbor classifier, for fricative classification. Modulation spectrogram gives good classification accuracy for recognition of place of articulation of fricatives and phoneme-level fricative classification. Feature vector dimension of $3 \times 3$ after applying HOSVD theorem is considered as optimum. Modulation frequency parameter plays more important role as compared to acoustic frequency parameter, as it affects the classification accuracy more significantly. One of the limitations of present work is that, we have worked on manually segmented fricative segments. In future, we would like to extend this work on continuous speech.

Acknowledgments. The authors would like to thank Department of Electronics and Information Technology (DeitY), New Delhi (India) and authorities of DA-IICT, Gandhinagar for their support to carry out this research work. We would also like to thank Mr. Maulik Madhavi for his support in the preparation of the paper.

\section{References}

1. Quatieri, T.F.: Discrete-time Speech Signal Processing: Principles and Practice. Prentice Hall Press, Upper Saddle River (2004)

2. Web Source, http://www.langsci.ucl.ac.uk/ipa/IPA_chart_c2005. pdf (last accessed on 30th April, 2013)

3. Garofolo, J.S.: Getting started with the DARPA TIMIT CD-ROM: An acoustic phonetic continuous speech database. National Institute of Standards and Technology (NIST), Gaithersburgh, MD (1988)

4. Scanlon, P., Ellis, D., Reilly, R.: Using Broad Phonetic Group Experts for Improved Speech Recognition. IEEE Trans. on Audio, Speech and Language Proc. 15, 803-812 (2007)

5. Ali, A.M.A., Spiegel, J.V., Mueller, P.: Acoustic-phonetic features for automatic classification of fricatives. J. Accoust. Soc. of America 109(5), 2217-2235 (2001)

6. Ali, A.M.A., Spiegel, J.V., Muller, P.: An acoustic-phonetic feature-based system for the automatic recognition of fricative consonents. In: IEEE Proc. on Int. Conf. on Acoustics, Speech and Signal Processing, vol. 2, pp. 961-964 (1998)

7. Seneff, S.: A Joint Synchrony/ Mean Rate Model of Auditory Speech Processing. J. Phonetics 16, 55-76 (1988)

8. Atlas, L., Shamma, A.S.: Joint acoustic and modulation frequency. EURASIP J. on Applied signal Proccessing 7, 668-675 (2003)

9. Greenberg, S., Kingsbury, B.: The modulation spectrogram: In pursuit of an invariant representation of speech. In: IEEE Proc. on Int. Conf. on Acoust., Speech, Signal Process., Munich, Germany, vol. 3, pp. 1647-1650 (1997)

10. Markaki, M., Stylianou, Y.: Voice pathology detection and discrimination based on modulation spectral features. IEEE Trans. on Audio, Speech, and Language Proc. 19(7), 1938-1948 (2011)

11. Lathauwer, L.D., Moor, B.D., Vandewalle, J.: A multilinear singular value decomposition. SIAM J. Matrix Anal. Appl. 21(4), 1253-1278 (2000)

12. Modulation Toolbox, http://www.ee.washington.edu/research/isdl/ projects/modulationtoolbox (last accessed on 30th April 2013) 